\title{
Modelling capillary barriers
}

\author{
J. Mls ${ }^{1}$ \& D. Trpkošová ${ }^{2}$ \\ ${ }^{1}$ Charles University in Prague, Czech Republic \\ ${ }^{2}$ Department of Chemistry of Fuel Cycle and Waste Management, \\ ÚJV Řež, Czech Republic
}

\begin{abstract}
When predicting the behaviour of a proposed capillary barrier, it is possible to utilize either the experimental measurements or the numerical modelling. Results of tipping trough modelling of a capillary barrier are used to study the reliability of numerical modelling. The required hydromechanical parameters of the capillary barrier layers are determined and used for the numerical repeating of the tipping trough experiments. The results are presented and discussed.

Subsequently, the paper studies the problem of capillary barriers efficiency. A criterion is introduced that makes use of soil-moisture retention data in order to predict the efficiency of a capillary barrier. Hysteresis of retention curves of the applied materials is studied and its effect on the efficiency is discussed.

Keywords: capillary barrier, retention curve, hysteresis, hydraulic characteristics, tension apparatus.
\end{abstract}

\section{Introduction}

A capillary barrier is one of methods used to close landfills. It is a relatively simple device that is built of two inclined layers of specially chosen materials. The upper layer, called the capillary layer, consists of a finer material, while the underlying layer, known as the capillary block, consists of a coarser material. The material of the capillary layer is mostly fine to medium sand and that of the capillary block is a uniform coarse-grained sand or fine-grained gravel. The device is aimed at protecting the landfill against a possible water infiltration.

The insulating effect of the capillary barrier is based on the fact that, under conditions of sufficiently low values of pressure head, the hydraulic conductivity of the capillary layer is at least by one order higher than that of the capillary block. 
If so, the water that enters the capillary barrier from above flows along the capillary block downwards rather than coming through the interface into the capillary block, e.g. [1-3]. As the amount of water kept in the capillary layer increases in the direction of its flow, it is necessary to find a safe length, the diversion length, after which the water has to be drained out of the layer, see $[1,4]$.

There is another kind of capillary barriers known as the combined capillary barriers, see [3]. These barriers contain a third layer that separates the capillary layer from the capillary block. The additional layer is usually a water repulsive geomembrane. In order to distinguish, the two-layers barriers are also called the simple capillary barriers. The reason for the double protection is in the fact that the simple barrier takes over the role of the geomembrane in the case of its failure [5].

When designing a capillary barrier, it is necessary to predict its function. There are two possibilities to do so. The first one is to use the supposed materials of the barrier and to make its physical model, e.g making use of a laboratory tipping trough. In this case one can test the behaviour of the proposed barrier under various values of inclination and various distributions of irrigation. The other possibility is to make use of mathematical modelling. It means to make use of a numerical model of unsaturated flow in porous media. This is a standard approach, see e.g. [5-9]. The crucial problem, in this case, is to properly determine all the involved parameters. Among them, the geometry of the flow domain can be easily define with any required accuracy. Similarly, the initial and boundary conditions, complicated as they may be, can be prescribed with any required accuracy. The most difficult problem is to determine hydromechanical parameters of the applied materials. The question on reliability of numerical modelling of capillary barrier is actually question on the ability to determine the material characteristics.

Having a reliable model of a given barrier, either physical or mathematical, one can find the answer to particular questions like what would be the diversion length, the effect of a slope change etc. On the other hand, modelling changes of the barrier's materials requires to completely rebuild the physical model or, when using a numerical model, to determine hydromechanical parameters of the new materials.

This paper presents results of a study concerned with both, the reliability of capillary barriers modelling and their efficiency. In order to test the agreement between numerical results and experimental measurements, the data obtained during a laboratory testing of two capillary barriers carried out at the Bochum University are utilized, see below.

\section{The applied materials}

Two different capillary barriers were investigated at the hydrogeological laboratory of the Ruhr-University in Bochum. The research was carried out in a large tipping trough and was aimed at testing two different couples of materials. The materials will be denoted by L1, L2, B1, B2 in this paper, with respect to the way of their utilization in the barriers, see also [9]: 


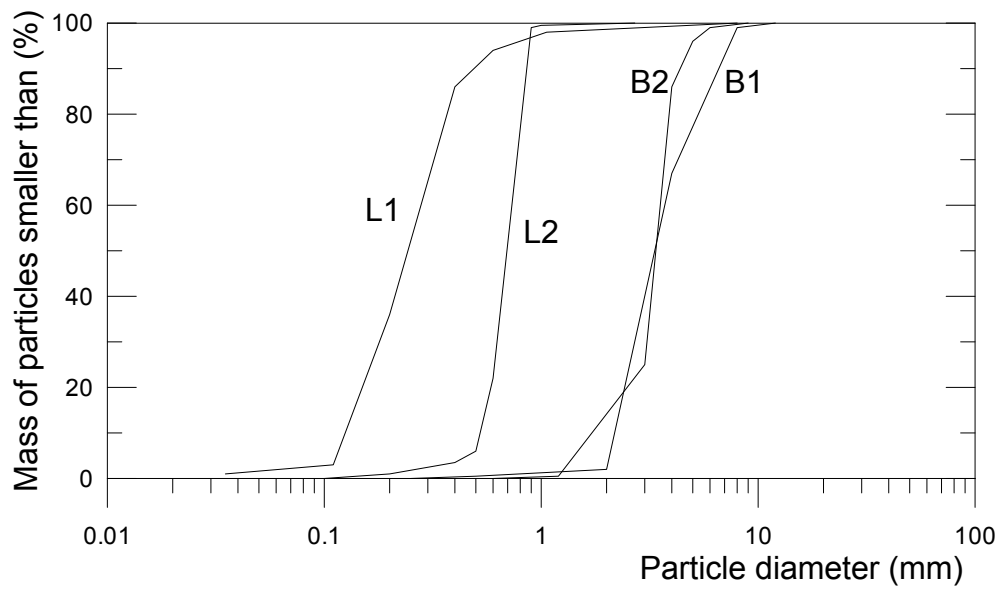

Figure 1: Grain-size curves of the applied materials.

B1: capillary block of the first barrier. It is a homogeneous material of grain size from $2 \mathrm{~mm}$ to $8 \mathrm{~mm}$. According to classification [10], it belongs to the category subrounded; the corners of grains are well rounded, their edges are partly rounded. The material is a product of the German company G2.

B2: capillary block of the capillary second barrier. It is a homogeneous material of grain size from $2 \mathrm{~mm}$ to $7 \mathrm{~mm}$. According to classification [10], it belongs to the category not rounded.

L1: capillary layer of the first barrier. It is a heterogeneous material originating in a river bed, from which the rough grains and calcareous layers were removed. According to classification [10], the sand belongs to the category rounded; both the corners and the edges of the grains are well rounded. The material is a product of the German company Tecklenborg.

L2: capillary layer of the second barrier. It is a homogeneous material of grain size from $0.4 \mathrm{~mm}$ to $1 \mathrm{~mm}$. According to classification [10], the sand belongs to the category rounded; both the corners and the edges of grains are well rounded.

The grain-size curves of the materials are shown in Figure 1. The grain-size data utilized in the figure were measured at Ruhr University in Bochum.

Wohnlich [11] presents just one hydromechanical characteristic that was determined at Bochum University - retention curve of one of the four materials. As it is not enough for numerical modelling of the barriers, it was necessary to determine complete set of hydromechanical characteristics by means of laboratory measurements carried out on samples of the materials. This was done at Charles University. The materials were obtained from the laboratory in Bochum. 


\section{Laboratory determination hydromechanical characteristics of the applied materials}

Water flow in the unsaturated zone is governed by the Richards equation. There are situations in capillary barriers where nonnegative values of pressure head are reached, e.g. when simulating drains. To be capable of solving such problems, the capacity form of the Richards equation has to be applied

$$
C(h) \frac{\partial h}{\partial t}=\frac{\partial}{\partial x_{i}}\left(K(h) \frac{\partial h}{\partial x_{i}}+\delta_{i, 3} K(h)\right),
$$

where $x=\left(x_{1}, x_{2}, x_{3}\right)$ are the space coordinates with the $x_{3}$-axis oriented vertically upwards, $t$ is time, $h(x, t)$ is the pressure head, $K(h)$ is the unsaturated hydraulic conductivity, and $C(h)$ is the capacity function,

$$
C(h)=\frac{\partial \theta}{\partial h}(h),
$$

where $\theta(h)$ is the retention curve.

As the dependence of hydraulic conductivity on the pressure head can also be determined from parameters of the corresponding retention curve, it was necessary to get the soil moisture retention data of the four materials. The method of tension apparatus was chosen for laboratory measurements as it was more convenient for the measured materials than the colon method or the pressure-plate apparatus. Moreover, the tension apparatus can be utilized for measurements in both directions, drying and wetting, without any modifications.

Samples of the four materials utilized in the capillary barriers in Bochum were taken to the laboratory of Soil mechanics of Charles University. A set of 18 samples, at least three of each material, was investigated. The size of the samples varied between $50 \mathrm{~cm}^{3}$ and $100 \mathrm{~cm}^{3}$. Both, the main drying branch and the main wetting branch were measured on each sample. Sets of 10 to 15 couples $(h, \theta)$ of the retention data were obtained on each sample and each branch. The particular points of the retention curves were measured in steps controlled by setting the values of pressure head.

Any measured sample was saturated initially and the main drying branch was measured. After reaching the residual saturation, the measurement of the main wetting branch started.

Another set of samples was utilized to measure saturated hydraulic conductivities of the tested materials. The samples were cylindrical, their diameter was $5 \mathrm{~cm}$ and their hight was $7 \mathrm{~cm}$. At least three samples of each material were measured and at least five measurements on each sample were carried out. The applied equipment was a permeameter working with constant differences of the hydraulic head at the ends of the measured sample.

As numerical solutions of problems with Equation (1) require to define retention curve for all admissible values of pressure head, the van Genuchten form

$$
\theta(h)=\theta_{r}+\frac{\theta_{s}-\theta_{r}}{\left(1+(-\alpha h)^{n}\right)^{m}},
$$


of the retention curve was chosen, where $h$ is pressure head, $\theta$ is water content, $\theta_{r}$ and $\theta_{s}$ are residual and saturated water contents, and $\alpha, n$ and $m$ are parameters satisfying $\alpha>0, n>1, m \in(0,1)$. It is usually required that $m=1-1 / n$; this relation is accepted also in the present paper. The parameter $\theta_{r}$ is unique for both branches of retention curves. As the relations between water content and pressure head depend on the direction of the process, there are generally different values of water content at saturation: $\theta_{s}^{d}$ for the main drying branch and $\theta_{s}^{w}$ for the main wetting branch. And similarly the parameters $\alpha^{d}, n^{d}$ of the drying branch differ from $\alpha^{w}, n^{w}$ of the wetting branch.

The Mualem model, based on statistics of pore-size distribution [12], was used in combination with Equation (2) to express the dependence of unsaturated hydraulic conductivity on pressure head:

$$
K(h)=\frac{K_{s}}{\sqrt{\left(1+(-\alpha h)^{n}\right)^{m}}}\left(1-\left(1-\frac{1}{1+(-\alpha h)^{n}}\right)^{m}\right)^{2},
$$

where $K_{s}$ is saturated hydraulic conductivity and $\alpha, m, n$ are the parameters introduced in Equation (2).

While the first parameters $\theta_{s}^{d}, \theta_{r}$ and $\theta_{s}^{w}$ were measured directly on samples, the remaining parameters were obtained by an optimization method. Particularly the code RETC by van Genuchten, Leu and Yates was utilized, see [13] to obtain the values $\alpha^{d}, n^{d}, \alpha^{w}$ and $n^{w}$. The complete set of parameters $\theta_{s}^{d}, \theta_{r}, \theta_{s}^{w}, \alpha^{d}, n^{d}, \alpha^{w}$, $n^{w}$ and $K_{s}$ is presented in Table 1 .

Table 1: Hydromechanical parameters of the four tested materials.

\begin{tabular}{lllllllll}
\hline & $\begin{array}{l}K_{s} \\
(\mathrm{~m} / \mathrm{s})\end{array}$ & $\theta_{r}$ & $\theta_{s}^{d}$ & $\begin{array}{l}\alpha^{d} \\
\mathrm{~cm}^{-1}\end{array}$ & $n^{d}$ & $\theta_{s}^{w}$ & $\begin{array}{l}\alpha^{w} \\
\mathrm{~cm}^{-1}\end{array}$ & $n^{w}$ \\
\hline B1 & $2.25 \mathrm{e}-3$ & 0.07 & 0.41 & 0.29 & 4.56 & 0.41 & 0.32 & 4.17 \\
B2 & $1.72 \mathrm{e}-3$ & 0.06 & 0.40 & 0.25 & 4.50 & 0.39 & 0.29 & 4.05 \\
L1 & $1.18 \mathrm{e}-4$ & 0.04 & 0.35 & 0.03 & 7.39 & 0.31 & 0.04 & 5.24 \\
$\mathrm{~L} 2$ & $7.63 \mathrm{e}-4$ & 0.02 & 0.41 & 0.06 & 4.45 & 0.38 & 0.09 & 3.23 \\
\hline
\end{tabular}

Main drying branches of retention curves of all the tested materials and main wetting branches of both capillary layers are shown in Figure 2. It is not necessary to show also the wetting branches of the capillary block materials as they are very close to the corresponding drying branches.

Hydraulic conductivities of the four materials as functions of pressure head are presented in Figure 3. The figure shows that there are regions of pressure head, $h<-4 \mathrm{~cm}$ for the first barrier and $h<-3 \mathrm{~cm}$ for the second barrier, 


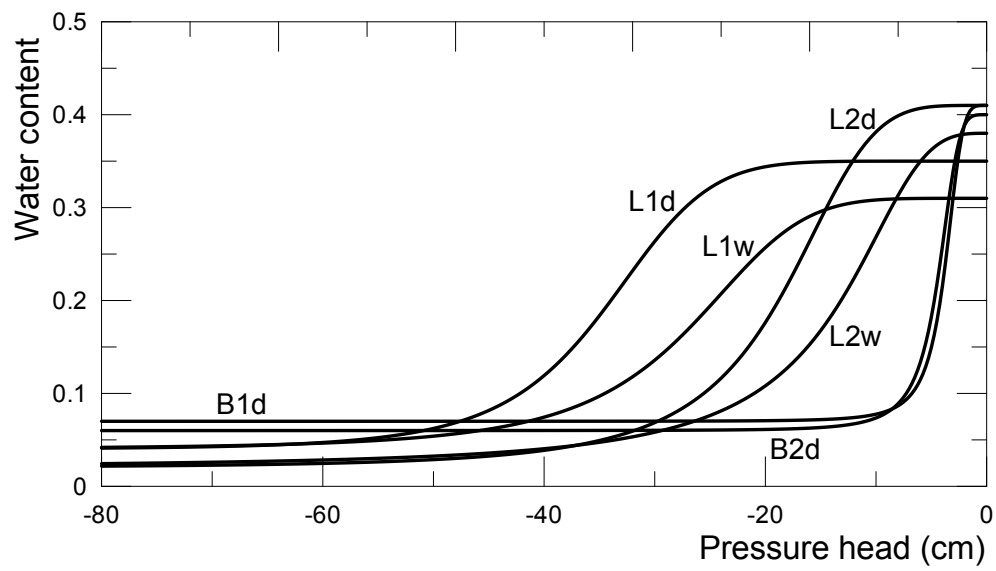

Figure 2: Retention curves of the applied materials.

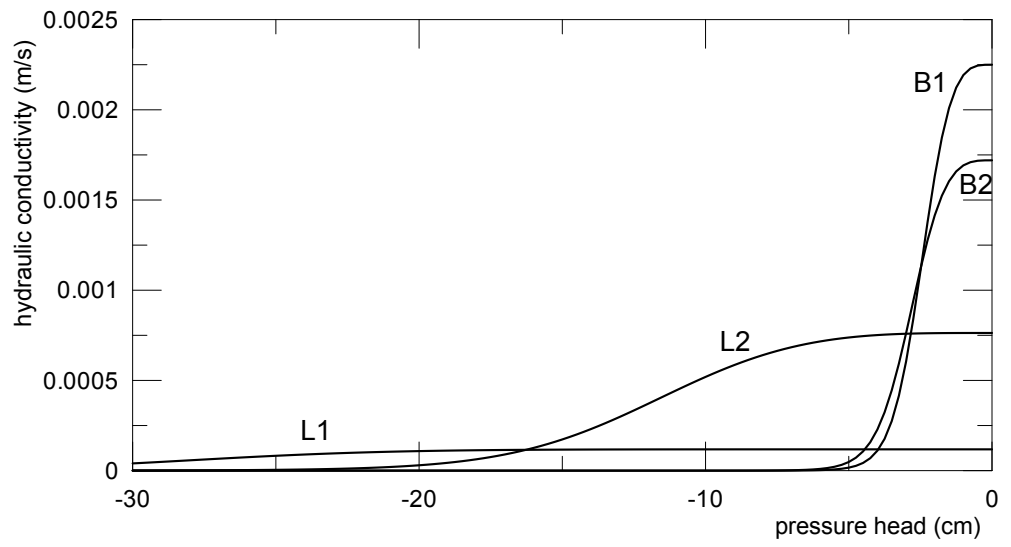

Figure 3: Hydraulic conductivities of the applied materials.

where hydraulic conductivities of the capillary layers are higher than those of the capillary blocks.

Several models were developed in order to incorporate the effect of hysteresis into the soil-moisture retention relations. The models are either empirical or theoretical, see [14]. Having obtained a complete set of the retention curve parameters $\theta_{s}^{d}, \theta_{r}, \theta_{s}^{w}, \alpha^{d}, n^{d}, \alpha^{w}, n^{w}$, hysteresis of retention curves can be described e.g. by means of the empirical model by [15]. It is convenient to 
introduce several assumptions. While $\theta_{r}^{d}=\theta_{r}^{w}=\theta_{r}, n^{d}=n^{w}=n$ and $\alpha^{d}<\alpha^{w}$ are accepted generally, Dohnal et al. [14] suggest to assume also $\theta_{s}^{d}=\theta_{s}^{w}=\theta_{s}$ in order to obtain the hysteresis loop closed also at the point of saturation. Figure 2 shows that, at least in the case of our soils CL1 and CL2, it is not desirable to force the equality $\theta_{s}^{d}=\theta_{s}^{w}$.

The curves depicted in Figure 2 and Figure 3 differ from those depicted in [9], where averaged values $n=n^{d}=n^{w}$ was used. Figure 2 and Figure 3 show functions $\theta(h)$ and $K(h)$ for the original values $n^{d} \neq n^{w}$ as they were obtained from the measured data.

\section{Numerical modelling of the tipping trough tests}

Numerical simulations represent an alternative to experimental measurements when examining the performance of the studied capillary barrier. One of the targets of this paper is to answer the question whether the numerical modelling can substitute for the laboratory experiments.

Two of the tipping trough tests carried out at the Ruhr University were repeated numerically. The geometry of the capillary layers was known as well as the space and time distribution of the applied irrigation. The hydromechanical characteristics presented in Table 1. were used to define parameters required in Equation (1).

Hysteresis is usually neglected in numerical simulations, e.g. [7] or [4]. On the other hand, Morris and Stormont [6] suggest that the pressure head and water flow conditions in the capillary barriers can be significantly affected by hysteresis. The numerical simulations presented in this paper were carried out using just the main drainage branches of the applied materials. The influence of hysteresis (and more generally of the moisture retention characteristics) was investigated separately.

The code S2D_dual [16] was used for the numerical modelling of the tipping trough experiments. The code solves the capacity form (1) of the Richards' equation using the finite element method. The M_GS code of the Menhart package [17] was used to generate the meshes of triangular elements that were required by the numerical method and the solved problems. Due to the discontinuity of the material parameters, it was necessary to make the mesh much denser at the capillary interface, where the distances of nodes shrank down to few millimeters.

A comparison of measured and computed results is shown in Figure 4. In the figure, the results of an experiment carried out with the first barrier are presented (see also [5], where the combined barriers were studied and these results were utilized in order to verify the model for its application to holes in the artificial sheeting). The period of the laboratory measurements was 6 weeks. The curve denoted by ir depicts the time distribution of the applied irrigation. The measured discharges from the capillary layer and from the capillary block are denoted by $\mathbf{L m}$ and Bm. The curves denoted by $\mathbf{L c}$ and Bc depict the computed discharge from the capillary layer and the capillary block respectively.

There is evidently a very good agreement between both couples $m$ and $c$ of curves expressing the discharge. The computed values are within the margin of error. The biggest differences at the beginning of the studied process are 


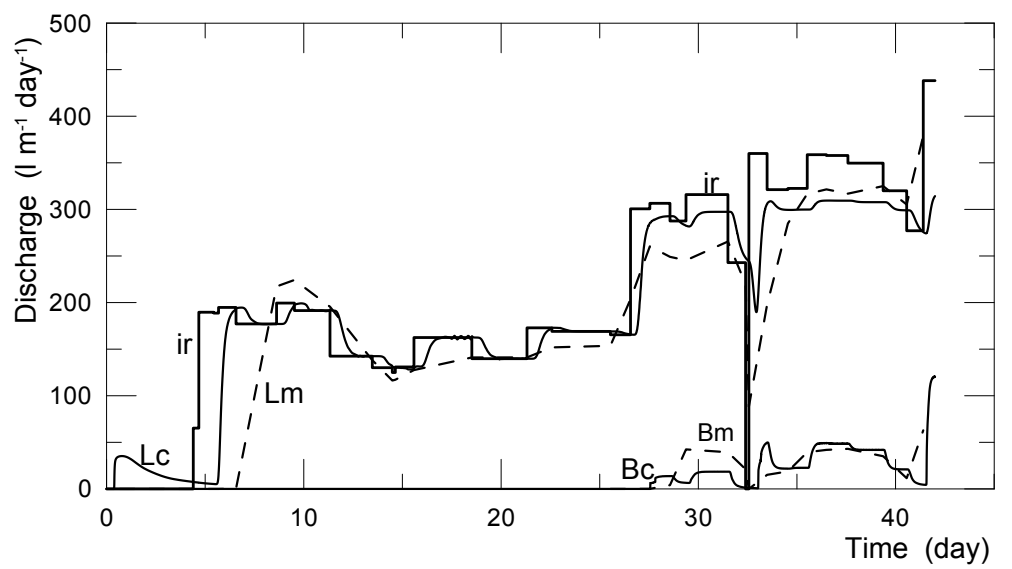

Figure 4: Tipping trough experiment, barrier No. 1; measured (m) and computed (c) discharge.

evidently due to the chosen initial moisture distribution. The imposed initial condition overestimated the real initial state and the numerical model indicates some discharge caused be the excess of water content in the capillary layer before the beginning of irrigation.

The intensity of the applied irrigation was set sufficiently large during the laboratory tests in order to break the insulation capacity of the barrier. It can be seen in the Figure 4 that this happened approximately 28 days after the begging of the test and that the measured and the computed data indicate almost identical starting time of the leakage.

\section{Reliability of capillary barriers}

The laboratory tests of the second barrier showed that there was some leakage through the capillary interface from the very beginning of the experiments. The efficiency of the second barrier was much worse than that of the first one. This fact may be surprising when comparing unsaturated hydraulic conductivities of the four applied materials, see Figure 3. The comparison suggests that the second barrier should be even better than the first one. In order to predict the efficiency of a barrier, it is necessary to consider not only the hydraulic conductivity but also retention curves.

The capillary layer has to contain a significant amount of water and to transfer it out of the protected region. Wang et al. [18] define the air entry value and the water entry value as pressure head at which the derivative $\theta^{\prime}(h)$ reaches its maximum value. When absorbing the infiltrated water, the pressure head values in 


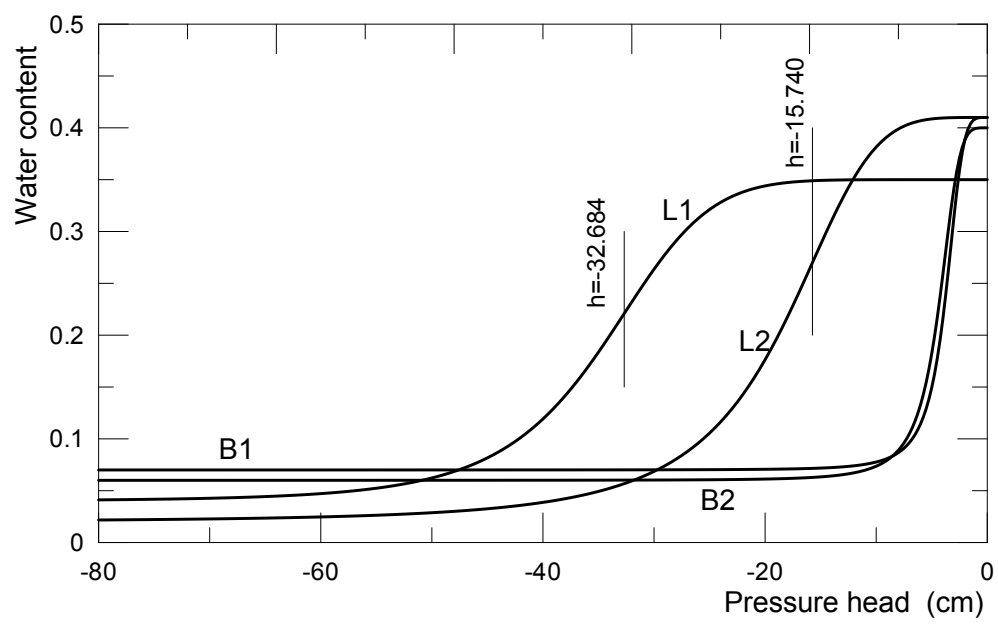

Figure 5: Drying branches of the retention curves of the four materials. Water entry values of both capillary layers are denoted by the vertical lines.

the capillary layer are equal to or higher than the water entry value. It follows from the continuity of pressure head that its values in the capillary block are close to those in the capillary layer. At the same time, these values of pressure head must be sufficiently below the water entry value of the capillary block. Hence, to be efficient, a capillary barrier should consist of materials whose water entry values are sufficiently distant from each other, see also [9].

In the Figure 5, drying branches of retention curves of the two barriers are depicted. Two vertical lines show the positions of the inflection points of the capillary-layer curves. The water entry values are added. The efficiency of a capillary barrier requires a sufficiently large pressure head interval between the water entry values of its two layers. The figure shows the significant difference between the lengths of the intervals of the barriers. This explains the fact that the efficiency of the first barrier is better than that of the second one. As a capillary barrier is broken during a process of increasing water content, the wetting branch of the capillary-layer retention curve should be considered rather than its drying branch. Consequently, see Figure 2, the length of the interval between the water entry values is actually even smaller than when using the drying branches.

\section{Conclusions}

Experimental results were used to study the reliability of numerical modelling of capillary barriers. To do this, the required hydromechanical parameters were 
obtained making use of soil moisture retention data measured on samples of the capillary barrier materials.

Two different tipping trough experiments were simulated numerically and the results were compared with measured data. It can be concluded that there is a good agreement between both data sets which suggests that numerical modelling can substitute for tipping trough measurements. It should be stressed that the numerical model was not calibrated to the experiments and the model parameters were obtained from measurements on samples of the applied materials.

The soil-moisture retention data were further utilized when studying the reliability of capillary barriers. A criterion based on the water entry values of the capillary barrier layers was introduced. The criterion shows, moreover, that the wetting branch of the capillary layer retention curve should be preferred to its drying branch, when studying the efficiency of the barrier. It was shown that the efficiency of a studied capillary barrier could be overestimated when neglecting hysteresis of its materials.

\section{Acknowledgements}

The research has been supported by the Grant Agency of the Czech Republic under grant No. 210/12/2451 and by the Ministry of Education of the Czech Republic under Research Plan No. MSM0021620855. The authors are also indebted to Prof. $\mathrm{S}$. Wohnlich for samples of the applied soils and for data utilized in Figures 1 and 4 .

\section{References}

[1] Ross, B., The diversion capacity of capillary barriers. Water Resources Research, 26(10), pp. 2625-2629, 1990.

[2] Picha, P., Laboratory testing of the capillary barrier. Acta Universitatis Carolinae Geologica, 48(1), pp. 29-36, 2004.

[3] Sehrbrock, U., Verbundsysteme aus kapillarsperre, dichtungsbahnen und geotextilien für die oberflächenabdichtung von deponien. 21. FachtagungDie sichere Deponie, Sicherung von Deponien und Altlasten mit Kunstoffen, Würzburg, 14p, 2006.

[4] Webb, S.W., Generalization of Ross' tilted capillary barrier diversion formula for different two-phase characteristic curves. Water Resources Research, 33(8), pp. 1855-1859, 1997.

[5] Trpkošová, D. \& Mls, J., The influence of artificial sealing on the capillary barrier's function. Waste Management, 30(1), pp. 125-131, 2010.

[6] Morris, C.E. \& Stormont, J.C., Evaluation of numerical simulations of capillary barrier field tests. Geotechnical and Geological Engineering, pp. 201-213, 1998.

[7] Oldenburg, C.M. \& Pruess, K., On numerical modeling of capillary barriers. Water Resources Research, 29(4), pp. 1045-1056, 1993. 
[8] Rudakov, D.V., Wohnlich, S. \& Barth, C., Numerical modeling of capillary barrier performance under growing infiltration. New approaches characterizing groundwater flow, vols 1 and 2, ed. Seiler, K. P. and Wohnlich, S., pp. 391-394, 2001.

[9] Trpkošová, D. \& Mls, J., Efficiency of capillary barriers in relation to retention curves data. Acta Geodynamica et Geomaterialia, 7(2), pp. 201207, 2010.

[10] Powers, M.C., A new roundness scale for sedimentary particles. Journal of Sedimentary Research, 23(2), pp. 117-119, 1953.

[11] Wohnlich, S., Untersuchungsbericht-Dichtigkeitsnachweis der Kombikapillardichtung (KKD). 3. Kipprinnenversuch, Bochum, 2006.

[12] Mualem, Y., New model for predicting hydraulic conductivity of unsaturated porous-media. Water Resources Research, 12(3), pp. 513-522, 1976.

[13] van Genuchten, M.T., Leu, F.J. \& Yates, S.R., The RETC code for quantifying the hydraulic functions of unsaturated soils. EPA, California, 1991.

[14] Dohnal, M., Dušek, J. \& Vogel, T., The impact of the retention curve hysteresis on prediction of soil water dynamics. Journal of Hydrology and Hydromechanics, 54(3), pp. 258-268, 2006.

[15] Kool, J.B. \& Parker, J.C., Development and evaluation of closed-form expressions for hysteretic soil hydraulic properties. Water Resources Research, pp. 105-114, 1987.

[16] Vogel, T., Documentation of the S2D code - version 2.0. CTU Praha, 1999 (internal).

[17] Mls, J., Description of the M_GS_v01 code. UK Praha, 2002 (in Czech, unpublished).

[18] Wang, Z., Wu, L. \& Wu, Q.J., Water-entry value as an alternative indicator of soil water-repellency and wettability. Journal of Hydrology, 231(SI), pp. 76-83, 2000. 